\title{
Temporal Lobe Epilepsy Lateralization Based on MR Image Intensity and Registration Features
}

\author{
S. Duchesne ${ }^{1}$, N. Bernasconi ${ }^{1}$, A. Janke ${ }^{2}$, A. Bernasconi ${ }^{1}$, and D.L. Collins ${ }^{1}$ \\ 1 Montreal Neurological Institute, McGill Univ., Montréal, Canada \\ 2 Center for Magnetic Resonance, Univ. of Queensland, Brisbane, Australia
}

\begin{abstract}
In the context of MR imaging, explicit segmentation followed by stereologic volumetry of the hippocampus (HC) has been the standard approach toward temporal lobe epilepsy (TLE) lateralization of the seizure focus. The novelty of the method presented here resides in its analysis of characteristics of large, non-specific Volumes of Interest from T1 MRI data aiming to lateralize the seizure focus in patients with TLE without segmentation. For this purpose, Principal Components Analysis (PCA) of two image features are united to create a multi-dimensional space representative of a training set population composed of 150 normal subjects. The feature instances consist of grey-level intensity and an approximation of the Jacobian matrix of non-linear registration-derived dense deformation fields. New data for TLE subjects are projected in this space, under the assumption that the distributions of the projections of normal and patients are not identical and can be used for lateralization. Results are presented following PCA modeling of the left medial temporal lobe only for all subjects. It is shown that linear discriminant analysis of the eigencoordinates can be used to lateralize the seizure focus in TLE patients with a $75 \%$ accuracy. It is expected that adding a right temporal lobe model will improve lateralization results beyond those of $\mathrm{HC}$ volumetry.
\end{abstract}

Keywords: MR intensity, non-linear deformation fields, jacobian matrices, principal components analysis, linear discriminant.

\section{Introduction}

Temporal lobe epilepsy (TLE) is defined by seizures originating in the medial temporal lobe (MTL). The majority of TLE patients are resistant to anticonvulsant drugs but can be helped by surgery, providing the seizure focus can be identified and surgically removed. The advent of high resolution MRI has had a major impact on the presurgical evaluation of patients with refractory epilepsy and has been demonstrated to be particularly useful for lateralizing the epileptic focus as being left or right MTL in origin.

Hippocampal sclerosis, characterized by cell loss and gliosis, is one of the most common patterns of damage in TLE [1]. Anatomical MRI of patients with TLE can show obvious atrophy or altered signal intensity (decreased intensity on T1weighted MRI and increased intensity in T2-weighted MRI) of the hippocampus 
(HC). Volumetric MRI of the $\mathrm{HC}$ is capable of detecting volume reduction with much greater sensitivity than simple inspection of routine anatomical MRI [4. TLE lateralization is then performed on the basis of $\mathrm{HC}$ atrophy, but clearly such technique requires a priori segmentation of the hippocampus.

Recent observations in animal models [3] and in patients with TLE [2] however indicate that the epileptogenic zone is broad, suggesting that the substrate for seizure generation is distributed over a network including several other structures in the MTL. Therefore, we believe it is possible to design a classification technique aiming at lateralizing the epileptic focus in TLE which would use information from a large, non-specific Volume of Interest (VOI) centered on the MTL, rather than a regional approach centered on a structure such as the $\mathrm{HC}$ (see fig.1).

Our research hypothesis is that this region contains sufficient discriminatory information between image intensity distributions and image registration features of normal and patient populations to effectively lateralize the seizure focus in new TLE subjects. The goal of our research in this area is to evaluate this hypothesis by developing a data driven classifier which would serve to lateralize the seizure focus in TLE patients based solely on MR image features.

\section{Methods}

Our method can be summarized as follows. First, a multidimensional eigenspace is created by uniting the results from two distinct Principal Component analyses of the following data: (a) linearly registered intensity images of the VOI; and (b) an approximation of the determinant of the Jacobian matrix of the deformation field for the given VOI. The deformation fields are obtained by non-linear registration of the VOI with a common reference image. We create a normal, non-pathological eigenspace by using processed data from a large training group of normal subjects $(N=150)$. Secondly, VOIs from study subjects, including 51 additional normal controls and 87 patients, are projected in the multidimensional eigenspace created. The last step consists of linear discriminant analyses (LDA) which are used to lateralize (left vs right TLE) the study subjects, based on their expressed eigencoordinates. The more general classification case (normals vs TLE) can also be attempted.

\subsection{Input Data for Multi-dimensional Space Creation}

Two image features have been retained for modeling. The first one is the trace, denoted by script $\mathbf{t}$. It consists of rasterized vectors of an approximation of the determinant of the Jacobian matrix (trace) of a non-linear registration-derived deformation field of any given subject toward a common reference target.

Of all the possible measures one could employ to characterize this dense deformation field, one of the simplest indicators of morphological changes is the trace of the Jacobian of the displacement matrix. Our implementation of trace calculation was discussed in Janke et al. [10], using the unified statistical 


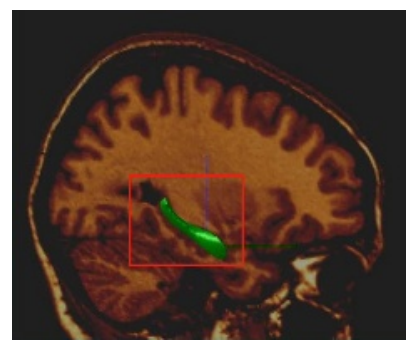

Fig. 1. Lateralization of TLE is commonly done using hippocampal volumetry (3D segmentation shown in green). Our approach, consistent with the growing evidence of extrahippocampal involvment in TLE, is to use intensity and registration information from a wider area (red box) centered on the medial temporal lobe
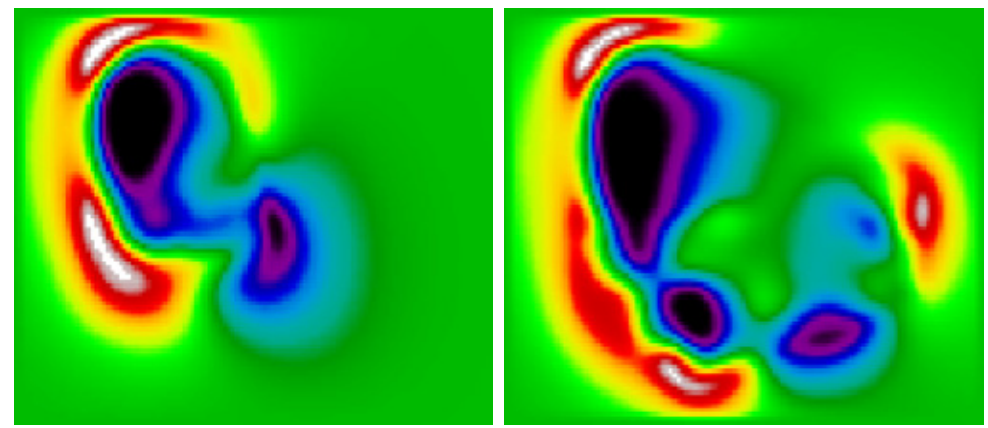

Fig. 2. (Left) Sagittal view through the medial axis of left trace volume for normal subject \#18. Green voxels do not move. From green to white (maximum) via yellows and reds indicate increase or expansion. From green to black (minimum), via blues and purples indicate decrease or contraction. The direction of movement is defined as the deformation which the subject's image must accomplish in order to align with the reference volume. Contraction represents atrophy in the case of tissue. (Right) Sagittal view through the medial axis of left trace volume for TLE subject \#10 with left hippocampal atrophy. Notice the contraction movement necessary to align the HC tail in the medium left quadrant, indicative of regional atrophy of structures in that area

framework proposed by Chung et al. [5]. If $\mathbf{U}$ is the displacement field which matches homologous points between two images, then the local volume change of the deformation in the neighborhood of any given voxel is determined by the Jacobian $J$ [5], which is defined as

$$
\mathbf{J}(x, t)=\operatorname{det}\left(\mathbf{I}+\frac{\delta \mathbf{U}}{\delta \mathbf{x}}\right)
$$

where $\mathbf{I}$ denotes an identity matrix and $\frac{\delta \mathbf{U}}{\delta \mathbf{x}}$ is the $3 \times 3$ displacement gradient matrix of $\mathbf{U}$ [5]. For relatively small displacements,

$$
\mathbf{J} \approx 1+\operatorname{tr}(\nabla \mathbf{U})
$$

as suggested by [5]. Hence the trace $\operatorname{tr}(\nabla \mathbf{U})$ represents a crude yet indicative measure of local volume change. This is well-suited to our application as the 
level of atrophy present between normal controls and TLE subjects is small yet significant and should therefore be apparent in the results of a local non-linear registration process. Examples are shown in Fig. 2

The second feature used in our classifier is intensity, denoted by script $\mathbf{g}$. Pre-processed images (see Sec. 3.1) serve as input to the model once difference volumes have been calculated. Those consist of the rasterized grey-level data from the pre-processed VOI $g$ subject input minus the average of all pre-processed volumes $g$ average input:

$$
\mathrm{g}=\mathrm{g} \text { subject input }-\mathrm{g} \text { average input }
$$

This average of 150 normal, healthy subject also forms the reference target for registration purposes (see Sec. 3.1). Readers should note that the PCA will thus be a zero-mean process modeling differences between subjects and the group mean. Original images and difference volumes are shown in fig. 3.

\subsection{Multi-dimensional Spaces, Features of Interest, and Classification}

Principal Components Analysis is used to reduce the dimensionality of the input data (intensity or trace) and generate a linear variation model. For the sake of brevity, the reader is encouraged to consult our mathematical description in [8],
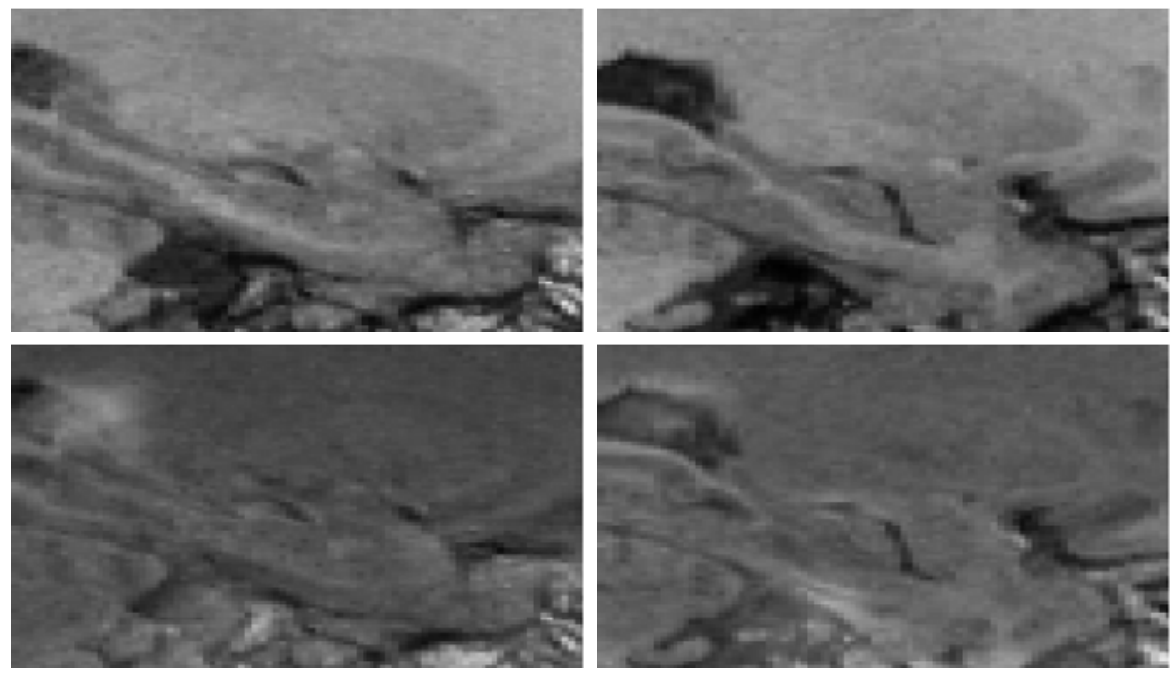

Fig. 3. (Top left) Sagittal view through the medial axis of left Volume of Interest (VOI) for normal subject \#18. (Top right) Sagittal view through the medial axis of left VOI for TLE subject \#10 with left hippocampal atrophy (HA). Notice the enlargement of the inferior horn of the lateral ventricle, indicative of tissue loss in this area. Likewise for the HC head. (Bottom left) Sagittal view through the left VOI medial axis of the difference image (difference $=$ original - mean) for normal subject \#18. As mentioned in the text, the PC analysis of intensity will therefore model deviations from the mean. (Bottom right) Same view but for TLE subject \#10 with left HA. 
itself based on the notation employed in Cootes et al. 7] in their work on Active Appearance Models.

The ensemble of Principal Components define an Allowable Grey Domain $\mathbf{G}$ as the space of all possible elements expressed by the grey-level intensity eigenvectors. Likewise, the Allowable Trace Domain $\mathbf{T}$ is the space of all possible elements expressed by trace eigenvectors

The theoretical upper-bound on the dimensionality of $\mathbf{B}$ and $\mathbf{T}$ is $f=N-1$ however we define restricted versions of these spaces, $\mathbf{G}^{*}$ with dimensionality $f_{G}$ and $\mathbf{T}^{*}$ with dimensionality $f_{T}$. Arbitrarily we have set $f_{G}=f_{T}=25$ for the current implementation of this technique. It is proposed to perform statistical analyses at a later date to determine the optimum number of eigenvectors which should be retained in each model yielding the best classifier performance.

Closely following the notation of Duda et al. 9], we have defined three states of nature $\omega$ for our study subjects: $\omega_{1}=$ normal, $\omega_{2}=$ left $H A$ and $\omega_{3}=$ right $H A$. Note that the 51 normal subjects are part of a control group and are not the same as the 150 subjects belonging to the training set used to develop the applicable Domains.

For the purposes of this work, the prior probabilities $p\left(\omega_{1}\right), p\left(\omega_{2}\right)$ and $p\left(\omega_{3}\right)$ are known since the compositions of the classification data sets are known exactly (see Sec. 3.1). They do not represent the normal incidence rates of TLE with left or right $\mathrm{HA}$ in the general population.

Pre-processed MRI intensity data (see Sec. 3.1) for each subject $i$ belonging to state $\omega$ are projected into the Domain $\mathbf{G}^{*}$ and thus form the eigencoordinate vectors $\gamma_{\mathbf{i}}^{\omega}$. Likewise, traces for each study subject are projected into the Domain $\mathbf{T}^{*}$ and thus form the eigencoordinate vectors $\tau_{\mathbf{i}}^{\omega}$.

A number of possible features can now be calculated on the distribution of the eigencoordinate vectors. The one we will originally base our classification scheme on is the position along the $\mathrm{PC}$ axis. We can reasonably assume that it can be represented by a Gaussian distribution, and thus the formulation of our feature vectors $\mathbf{p}$ for each subject $i$ follows easily:

$$
\mathbf{p}_{\mathbf{i}}^{\omega}=\gamma_{\mathbf{i}}^{\omega} \cup \tau_{\mathbf{i}}^{\omega}
$$

We have chosen to use a simple, multivariate linear discriminant analysis as our classifier. The more general case of subject classification into normals and TLE will be attempted first, followed by a lateralization attempt into left and right seizure focus for TLE patients only.

\section{Experiments and Results}

\subsection{Subjects and Image Preparation}

The training set consisted of $N=150$ subjects (mean age: 24.8 yrs; 86 males) from the International Consortium for Brain Mapping database (ICBM) [11.

The study population consisted of 87 consecutive patients with intractable, non-foreign-tissue TLE (mean age: 35 yrs; 47 males), and 51 neurologically normal controls (mean age: 33 yrs, 27 males). Lateralization of seizure focus in TLE 
patients was determined by a comprehensive evaluation including prolonged video-electroencephalogram (EEG) telemetry. The EEG focus was defined as right or left if more than $70 \%$ of seizures were recorded from one side. Manual MRI volumetry showed hippocampal atrophy ipsilateral to the seizure focus in all patients. 47 patients had left and 40 right hippocampal atrophy (HA).

MR 3D images were acquired on a $1.5 \mathrm{~T}$ scanner using a T1-fast field echo sequence. All global MRI data were pre-processed to correct for intensity nonuniformity due to scanner variations [12, linearly registered into stereotaxic space and resampled onto a $1 \mathrm{~mm}$ isotropic grid [6]. The Volume of Interest for this study was centered on the left medial temporal lobe $(55 \times 82 \times 80=360800$ voxels) for all subjects in the study. This should not be confused with the fact that some patients had predominantly left or right HA. The extent of this volume captured the hippocampus and neighboring MTL structures irrespective of normal inter- and intra-individual variability. The VOIs were then linearly registered (locally) onto the reference target mentioned earlier to further reduce positional variations which would propagate as unwanted noise in the morphometric PCA modelling. The reference target is the average of the 150 ICBM subjects that form part of the training set. This average was used to produce the difference volumes of eq. 3 . Finally, VOIs were intensity-normalized with respect to the reference target for the same reason.

The reader should note that in the intensity modeling, we did not use nonlinearly registered VOI for the simple reason that it would induce conformity in all data sets, thereby eliminating the very anatomical variability which we aim to model. The results from non-linear registration are solely used in the trace calculations.

\subsection{Creation of Multi-dimensional Spaces and LDA}

The method described above was used to build a model of left MTL intensity for which eigencoordinates along the first 25 eigenvectors for each model (25 intensity, 25 trace, based on the eigenpower fraction of total eigenpower) were chosen to to be presented to the classifier. Statistical analyses aiming at optimally selecting the number of eigenvectors to be retained in each model for classification will be the subject of future work.

Prior probabilities for each state of nature $\omega$ were not equal: $p_{\omega_{1}}=0.37, p_{\omega_{2}}=$ 0.34 and $p_{\omega_{3}}=0.29$.

Our multivariate linear discriminant analysis was done using SYSTAT 10.2 (Richmond, CA), with a tolerance of $F=0.001$. Fig. 4 displays boxplots of eigencoordinates for the 4 most discriminating eigenvectors, the first 3 being trace PCs and the last an intensity PC.

\subsection{Classification and Lateralization}

The first test of our system was the classification of subjects into normal $\left(\omega_{1}\right)$ or TLE $\left(\omega_{2}, \omega_{3}\right)$ states. Following backward stepwise regression, we were able 

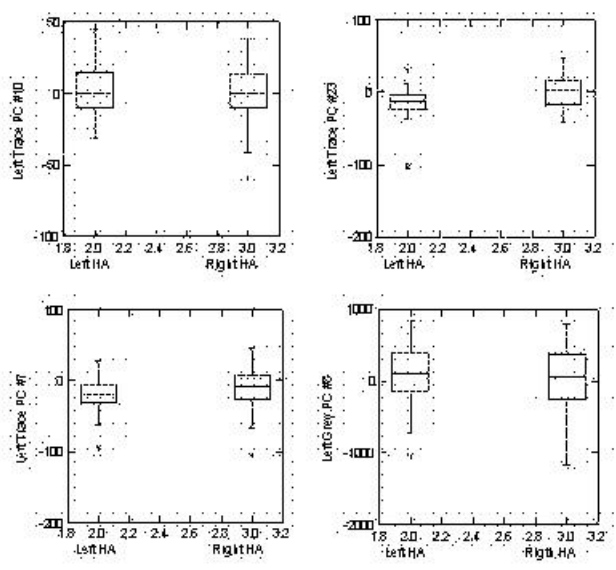

Fig. 4. Boxplot of eigencoordinates for the four most discriminating eigenvectors, the 3 most discriminating ones being trace PCs, followed by an intensity PC.

to obtain a classification rate of $95 \%$ using our intensity and trace models. 20 eigenvectors out of 50 were kept by the backward stepwise regression process.

Lateralization results are summarized in Table 2. Only TLE subjects were presented to the classifier, using the same data as above. The best lateralization results were obtained using forward stepwise regression with identical tolerance as previously. We obtain a lateralization for left vs right HA of $75 \%$.

Table 1. Left - Backward stepwise classification gives best results to classify subjects as belonging to state 1 (normals) or $2+3$ (TLE). True positive results on the NormalsNormals / TLE-TLE diagonal, shown in bold. Right - Forward stepwise classification gives best results to lateralize TLE subjects as belonging to state 2 (left HA) or 3 (right HA). True positive results on the LHA-LHA / RHA-RHA diagonal, shown in bold.

\begin{tabular}{cccccccccc} 
& Normals & TLE & $\%$ correct & & \multicolumn{3}{c}{ Left HA } & Right HA & \% correct \\
\cline { 1 - 3 } \cline { 5 - 7 } Normals & $\mathbf{4 5}$ & 6 & 88 & & Left HA & $\mathbf{3 6}$ & 11 & 77 \\
TLE & 1 & $\mathbf{8 6}$ & 99 & & Right HA & 11 & $\mathbf{2 9}$ & 73 \\
Total & 46 & 92 & $\mathbf{9 5}$ & & Total & 47 & 40 & $\mathbf{7 5}$
\end{tabular}

\section{Discussion}

The formulation of this classification problem is simple and flexible, allowing a number of possible features to be used without extensive reengineering. Our results indicate that the position information (eigencoordinates) of new data once projected in multidimensional Domains is sufficient to adequately discriminate between our two populations. Some impressive data reduction also takes place, as one starts from a model of 150 VOIs $\left(150 \times 360800\right.$ voxels $=5.4 \times 10^{6}$ points in MRI space) down to 50 data points in $\mathrm{PC}$ space. It is expected that adding information from a right-sided model will noticeably improve lateralization results. 
Future work will investigate the possibility of including the eigencoordinates of the projection of all training set data into a Bayesian-type classification scheme. We will also examine the behavior of the classifier when presented with more eigenvector information and different tolerance values in order to determine the optimal number of eigenvectors to retain in the modeling. Finally, the clinically-oriented members of our team will ponder the identification of the anatomical and pathological importance of the Principal Components holding maximum discriminatory power. It is expected that some PCs will give information as to the spatial distribution of pathological variations, information which can also be used in the surgical evaluation.

\section{Conclusion}

The underlying assumption for this work is that there exists sufficient information in a chosen VOI of normal and abnormal subjects MR global volumes to be used for the correct lateralization of seizure focus in TLE patients. Our results demonstrate that multidimensional spaces can be created from a PCA of extracted features of interest from training set images, and that such a space forms an adequate basis for the discrimination between subject projections.

Our results further indicate that LDA can identify and lateralize TLE with a high degree of success. The proposed methodology does not rely on segmentation, requires no user input and is data-driven. It is anticipated that such systems will gain widespread use in the next few years. The ability to lateralise TLE based solely on MR imaging has great potential to improve current diagnostic procedures.

Acknowledgments. This work was supported in whole or in part by FCAR, Manulife Financial / CCPE and CIHR. The authors would also like to thank ICBM for the right to use some data.

\section{References}

[1] T. Babb and W. Brown, pages 511-540. New York, NY: Raven, 1987.

[2] N. Bernasconi et al., Brain, 126(2): 462-469, 2003.

[3] E. Bertram, Epilepsia, 38(1): 95-105, 1997.

[4] F. Cendes et al., Ann Neurology, 42: 737-746, 1997.

[5] M. Chung et al., NeuroImage, 14(3): 595-606, 2001.

[6] D. Collins et al., IJPRAI, 11: 1271-1294, 1997.

[7] T. F. Cootes et al., IEEE PAMI, 23: 6,681-685, 2001.

[8] S. Duchesne et al., Proc. ICPR, 1: 520-523, 2002.

[9] R. Duda, P. Hart, and D. Stork. Pattern Classification. Wiley-Inter., 2nd ed., 2001.

[10] A. Janke et al., MRM, 46(4): 661-666, 2001.

[11] J. Mazziotta et al., NeuroImage, 2: 89-101, 1995.

[12] J. Sled et al., IEEE TMI, 17: 87-97, 1998. 